\title{
CHIP Enhances Angiogenesis and Restores Cardiac Function After Infarction in Transgenic Mice
}

\author{
Cheng-Wei Xu $u^{a}$ Tian-Peng Zhang ${ }^{b}$ Hong-Xia Wang ${ }^{b}$ Hui Yang ${ }^{b}$ Hui-Hua Lib \\ aDepartment of Laboratory Medicine, the Second Hospital of Shandong University, Jinan; ${ }^{\mathrm{b} T h e}$ \\ Key Laboratory of Remodeling-Related Cardiovascular Diseases, Department of Pathology and \\ Pathophysiology, School of Basic Medical Sciences, Capital Medical University, Beijing
}

\section{Key Words}

Carboxyl terminus of Hsp70-interacting protein - Myocardial infarction - Angiogenesis • Apoptosis • Inflammation • Cardiac dysfunction

\begin{abstract}
Background: Carboxyl terminus of Hsp70-interacting protein (CHIP) is a chaperone/ ubiquitin ligase that plays an important role in stress-induced apoptosis. However, the effect of CHIP on angiogenesis, cardiac function and survival 4 weeks after myocardial infarction (MI) remain to be explored. Methods: Wild-type (WT) and transgenic mice (TG) with cardiac-specific overexpression of CHIP were used for coronary artery ligation. The cardiac function, cardiomyocyte apoptosis, inflammation and angiogenesis were examined by echocardiography, histological analysis, real-time PCR and Western blot analysis. Results: At 4 weeks of after coronary artery ligation, echocardiography demonstrated that cardiac remodeling and dysfunction were prevented in TG mice compared with WT mice. The infarct size, cardiomyocyte apoptosis and inflammation were significantly reduced in TG mice than in WT mice. The survival rate after MI in TG mice was higher than that of WT mice. Furthermore, the levels of p53 protein was markedly decreased, but the expression of HIF- $1 \alpha$ and VEGF, and the formation of capillary and arteriole after MI were significantly enhanced in TG mice compared with WT mice. Conclusion: We report the first in vivo evidence that CHIP enhances angiogenesis, inhibits inflammation, restores cardiac function, and improves survival at 4 weeks after MI. The present study expands on previous results and defines a novel mechanism. Thus, increased CHIP level may provide a novel therapeutic approach for left ventricular dysfunction after MI.
\end{abstract}




\section{Introduction}

Heart failure (HF) is a consequence of the maladaptive cardiac remodeling that can be induced by various types of heart disease, such as chronic hypertension, myocardial ischaemia or infarction [1]. Myocardial infarction (MI) is an irreversible injury caused by the occlusion of a coronary artery, leading to cardiomyocyte death, infiltration of inflammatory cells, production of extracellular matrix and scar formation [1]; however, the exact mechanisms for these effects are not fully understood. It is well established that angiogenesis plays a critical role in various pathological settings, including tumor growth, wound repair and MI, and may promote cardiac growth and maintain contractile function [2]. Several angiogenic factors including vascular endothelial growth factor (VEGF), hypoxia-inducible factor (HIF1), p53 and others involve this process [2,3]. Recently, studies have demonstrated that heat shock proteins (HSPs) are abundantly expressed in myocardial cells and are important for myocardial function in response to stresses [4,5]. For example, heat shock protein 90 (HSP90) exerts pro-angiogenic effects through Akt and endothelial Nitric Oxide Synthase [4]. Overexpression of Hsp70 improves functional recovery and reduces infarct size after ischaemia through protection of mitochondrial function [5]. Thus, HSPs exert a critical role in cardioprotection during ischemic stress.

Carboxyl terminus of Hsp70-interacting protein (CHIP, also known as Stub1) has been identified as a dual-function cochaperone/ubiquitin E3 ligase that is highly expressed in a variety of mammalian tissues and cells, especially in the heart. CHIP interacts with Hsp70/ Hsp90 and targets chaperone-bound client proteins, including p53, ErbB2, cystic fibrosis transmembrane conductance regulator, Tau, Ask1, ataxin-1, Foxo1, and myocardin, for the ubiquitin-mediated degradation [6-14]. Numerous studies have demonstrated that CHIP plays an important role in preventing apoptosis in response to various stimuli [11, 13-18]. CHIP deficiency causes marked apoptosis in cardiomyocytes and endothelial cells of intramural vessels in response to ischaemia/reperfusion (I/R) injury [15]. In contrast, overexpression of CHIP inhibits ASK1-mediated apoptosis [11]. Moreover, our new data demonstrate that CHIP also inhibits angiotensin II (Ang II)-induced cardiac fibrosis and inflammation through inhibition of NF- $\mathrm{BB}$ and mitogen-activated protein kinase pathways [18]. Interestingly, a recent study confirm that overexpression of CHIP protects ischaemic apoptosis and cardiac injury through MDM2-mediated p53 degradation [16]. However, the effect of CHIP on cardiac dysfunction, survival and the precise mechanisms at 4 weeks after myocardial infarction (MI) remain to be investigated.

Based on these findings, the present study attempts to expand our understanding of the cardioprotective mechanisms of CHIP. We hypothesized that CHIP enhances angiogenesis and improves cardiac function after MI. To test this hypothesis, we used transgenic (TG) mice with cardiac-specific overexpression of CHIP under the a-myosin heavy chain (MHC) promoter and examined cardiac function, cardiomyocyte apoptosis, inflammation and angiogenesis at 4 weeks after coronary artery ligation. The present study expanded previous results and demonstrate that in addition to preventing p53-mediated cardiomyocyte apoptosis, CHIP also play an important role in inhibiting inflammation and enhancing angiogenesis, thereby leading to improvement of cardiac function and animal survival after MI.

\section{Materials and Methods}

\section{Transgenic mice}

Transgenic mice expressing CHIP in the heart were created as described [18]. Wild-type (WT) and CHIP transgenic mice (TG) male mice (10-12-week-old, C57BL/6 background) were anesthetized with $2 \%$ isoflurane inhalation and were then subjected to operation of myocardial infarction model by ligation of left coronary artery (LCA) as described [19]. The sham group underwent the same surgical procedure except that the LCA was not occluded. Mice were sacrificed at 28 day post-operation and heart tissues were harvested. All procedures were approved by the Animal Care and Use Committee of Peking Union Medical 
Xu/Zhang/Wang et al.: CHIP Improves Angiogenesis and Cardiac Dysfunction After Infarction

College. The investigation conformed to the Guide for the Care and Use of Laboratory Animals published by the US National Institutes of Health (NIH Publication No. 85-23, revised 1996).

\section{Echocardiography}

Echocardiographic measurement was performed with a Vevo 770 ultrasound system (VisualSonics Inc.) equipped with a 30-MHz transducer as described previously $[18,20]$. Fractional shortening (FS) was calculated as follows: FS=[(LVEDD-LVESD)/LVEDD]x100, where LVEDD is left ventricular end-diastolic diameter, and LVESD is left ventricular end-systolic diameter.

\section{Histological and Immunohistochemical analysis}

Histologic analysis of hearts from WT and transgenic mice were performed according to standard protocols. Sections $(5-\mu \mathrm{m})$ were deparaffinized and stained with hematoxyliny and eosin (H\&E), Masson's trichrome and wheat germ agglutinin-TRITC conjugate as described previously [21-23]. To identify infiltrating macrophage populations, sections were incubated with anti-Mac-2 (CD11b, clone M1/70) antibody (Abcam Inc., Cambridge, UK) or isotype control overnight at $4^{\circ} \mathrm{C}$, and then incubated with horseradish peroxidase-conjugated goat anti-rat antibody (Santa Cruz Biotechnology, Santa Cruz, CA). $[18,21-23]$. Isolectin staining was performed to identify capillaries according manufacturer's instructions. FITC-conjugated isolectin B4 (Vector Labs) was used as primary antibody without secondary antibody. Antia-smooth muscle actin (a-SMA) antibody (1:500; Abcam Cambridge, MA) was applied to identify a-SMApositive vessels [18].

\section{TUNEL assay}

Apoptosis was evaluated by terminal deoxynucleotidyl transferase-mediated dUTP nick end labeling (TUNEL) assay (Roche Diagnostic, Penzberg, Germany) in heart sections according to manufacturer's instructions as described [18]. The percentage of TUNEL-positive myocytes was determined by counting 10 random fields per section under a microscope (magnification, $\times 200$ ) (BX50, Olympus).

\section{Quantitative real-time PCR analysis}

Hearts from WT and TG mice were excised, rinsed in PBS, frozen in liquid nitrogen, and stored at $-80^{\circ} \mathrm{C}$. Total RNA was purified from intact hearts with Trizol (Invitrogen Corp.) as described previously [18]. The mRNA expression of Bax, Bcl-2, TNF- $\alpha$, IL-1 $\beta$, HIF-1 $\alpha$, VEGF and GAPDH (as a control) were measured by quantitative real-time PCR (qPCR) with primers as described previously $[18,20]$. The mRNA expression levels were normalized to GAPDH.

\section{Western blot analysis}

Protein samples were prepared from hearts using lysis buffer $(20 \mathrm{mM}$ Tris (pH 7.5), $150 \mathrm{mM} \mathrm{NaCl}, 1$ mM EDTA, $1 \mathrm{mM}$ EGTA, 1\% Triton X-100, $2.5 \mathrm{mM}$ sodium pyrophosphate, $1 \mathrm{mM} \beta$-glycerophosphate, $1 \mathrm{mM}$ $\mathrm{Na}_{3} \mathrm{VO}_{4^{\prime}} 4 \mu \mathrm{g} / \mathrm{ml}$ aprotinin, $4 \mu \mathrm{g} / \mathrm{ml}$ leupeptin, $4 \mu \mathrm{g} / \mathrm{ml}$ pepstatin, and $1 \mathrm{mM}$ PMSF). Western blot analysis was performed as described [24, 25]. Fifty $\mu$ g protein lysates were separated by $10 \%$ SDS-PAGE and then transferred to nitrocellulose membranes (Bio-Rad), the membranes were incubated with primary antibody against p53 (1:500), CHIP (1:500; Santa Cruz Biotechnology, Santa Cruz, CA) and $\beta$-actin (Cell Signaling Technology, Beverly, MA, 1:3000) at $4^{\circ} \mathrm{C}$ overnight. The levels of signaling proteins were normalized to the levels of $\beta$-actin. Protein levels were quantified by using Gel-pro 4.5 Analyzer (Media Cybernetics).

\section{Survival analysis}

The survival analysis was performed in WT and CHIP-TG $(n=11-24)$ mice after sham or MI. During the study period of 28 days, the mice were allowed free access to food and water. Cages were inspected daily for animals that had died. Survival of mice 28 days after MI was analyzed using Kaplan-Meier methods and the log-rank test.

\section{Statistical analysis}

Data are presented as means \pm SEM. Differences between WT and CHIP-TG mice at discrete time points were evaluated for statistical significance using Student's $t$ test or ANOVA. P values less than 0.05 were regarded as significant. 
Fig. 1. Transgenic expression of CHIP improves post-infarction cardiac remodeling and function. (A) Representative M-mode echocardiograms from wild-type (WT) and CHIP transgenic (TG) mice at 4 weeks after sham operation or myocardial infarction (MI). (B) Group echocardiographic data: LVEDV, LV end-diastolic volume; LVESD, LV endsystolic volume; LVFS, LV fraction shortening; and LVEF, LV ejection fraction. Data expressed as means \pm S. E. M (n=12 per group). ${ }^{*} P$ $<0.05$ vs. sham; ${ }^{\#} P<0.05$ vs. WT+MI.



\section{Results}

CHIP TG mice have increased cardiac function after MI

To investigate the physiopathologic role of CHIP in cardiac function, myocardial infarction (MI) was induced by ligation of left coronary artery (LAC) in WT and CHIP-TG mice. At 4 weeks after MI, cardiac function of WT and TG mice were examined by echocardiography. There was a significant chamber dilatation, as characterized by increased left ventricle (LV) end-diastolic volume (LVEDV ) and LV end-systolic volume (LVESD), and systolic dysfunction, as assessed by LV fractional shortening (FS\%) and ejection fraction (EF\%) in infarcted groups over sham. However, LV dilatation and dysfunction were attenuated in TG mice compared with WT mice after infarction (Fig. 1A and B). TG mice displayed similar cardiac size and function compared to WT animals at baseline (Fig. 1A and B). These data suggest that overexpression of CHIP preserves cardiac dilation and function after MI.

\section{Cardiac CHIP overexpression reduces infarct size and mortality after MI}

To determine whether overexpression of CHIP attenuates cardiac injury, we assessed the infarct size with 2, 3, 5-triphenyltetrazolium chloride (TTC) staining for the WT and TG hearts after IMI. TG mice had a lower percent of infarct size compared to WT mice (Fig. 2A). Furthermore, fibrotic areas were significantly lower in TG mice than WT mice as assessed by Masson trichrome staining (Fig. 2B). There was no difference in the infarct size and fibrotic areas between 2 groups after sham (Fig. 2A and B). Moreover, the survival rate up to 4 weeks after MI was significantly higher in TG mice than in WT mice ( $91.7 \%$ vs. $74.1 \%)$. There were no deaths in sham-operated groups (Fig. 2C). 
Fig. 2. CHIP overexpression reduces infarct size and improves survival. (A) Representative photographs of short-axis LV sections from WT and TG mice after sham or myocardial infarction (MI) (left). Bar graph shows the quantification of mean infarct size (right, $n=8$ per group). (B) Masson trichrome staining of the heart sections from WT and TG mice at 4 weeks after sham and myocardial infarction (MI) (left). Bar graph shows the quantification of fibrotic areas (right, $\mathrm{n}=8$ per group). Bar= $50 \mu \mathrm{m}$. (C) Kaplan-Meier survival curves in WT and TG mice up to 4 weeks after sham operation or myocardial infarction (MI) $(n=12$ 24 per group). Data expressed as means \pm S. E. M. ${ }^{*} P<0.05$ vs. sham; ${ }^{\#} P<0.05$ vs. WT + MI.

Fig. 3. CHIP overexpression inhibits cardiomyocyte apoptosis. (A) Representative photomicrographs of cardiac cell apoptosis in myocardial border area of WT and TG mice after sham or myocardial infarction (MI) examined by TUNEL assay (green). Myocytes were identified by immunostaining with anti- $\alpha$ actinin antibody (red), and nuclei were counterstained with DAPI (blue) (left). Bar graph shows the quantification of TUNEL-positive cells from WT and TG mice (right). (B) Ratio of Bax/Bcl-2 mRNA expression in the myocardial border area of WT and TG mice after sham or myocardial infarction (MI). Data expressed as means $\pm \mathrm{S}$. E. M. (n=8 per group). ${ }^{*} P<0.05$ vs. sham; ${ }^{\#} P<0.05$ vs. WT+MI.
A
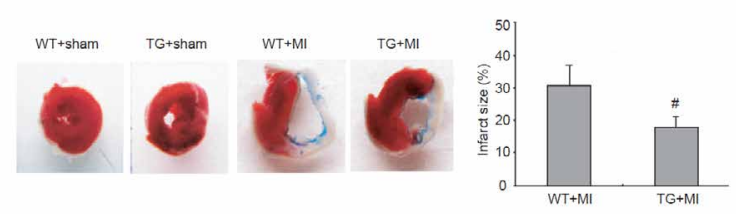

B
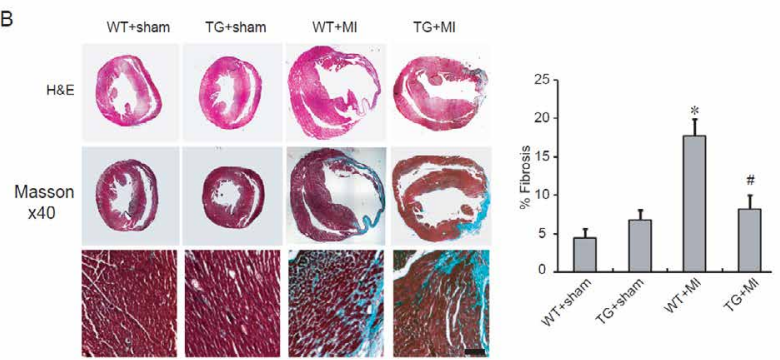

C

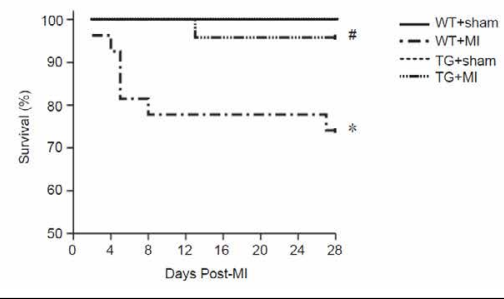

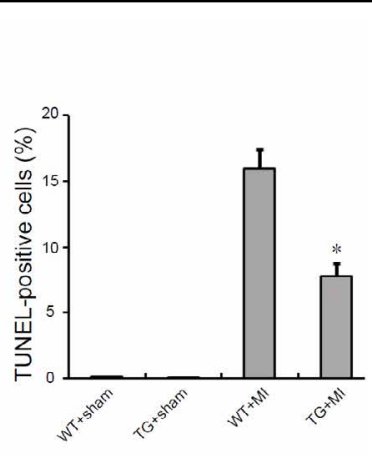

B
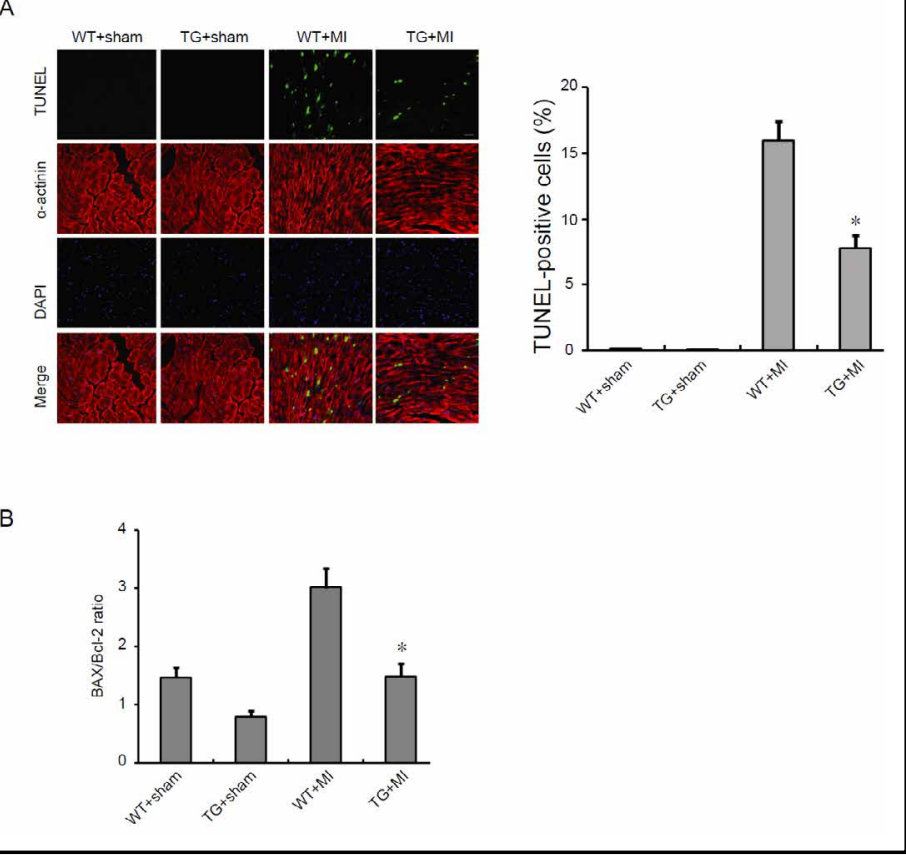

\section{1}

Overexpression of CHIP inhibits cardiomyocyte apoptosis

To assess whether CHIP affects cardiomyocyte apoptosis, we detected the number of apoptosis and the levels of Bax and Bcl2 mRNA expression in the heart tissue using TUNEL assay and qPCR analysis. As shown in Fig. 3A and B, TG mice displayed a significantly lower degree of TUNEL-positive cardiomyocytes and Bax/Bcl-2 ratio compared to WT mice. 
Fig. 4. CHIP overexpression attenuates myocardial inflammation. (A) Immunohistochemistry of the myocardial border area from WT and TG mice after sham and myocardial infarction (MI) for Mac-2 (a marker for macrophages) (left). Bar graph shows the quantification of Mac-2-positive macrophages (right, $n=8$ per group). (B) qPCR analysis of the mRNA levels of IL$1 \beta$ and TNF- $\alpha$ in the myocardial border area from WT and TG mice after sham and myocardial infarction (MI). Data expressed as means \pm S. E. M. ( $n=8$ per group). ${ }^{*} P<0.05$ vs. sham; ${ }^{*} P<0.05$ vs. WT+MI.
A

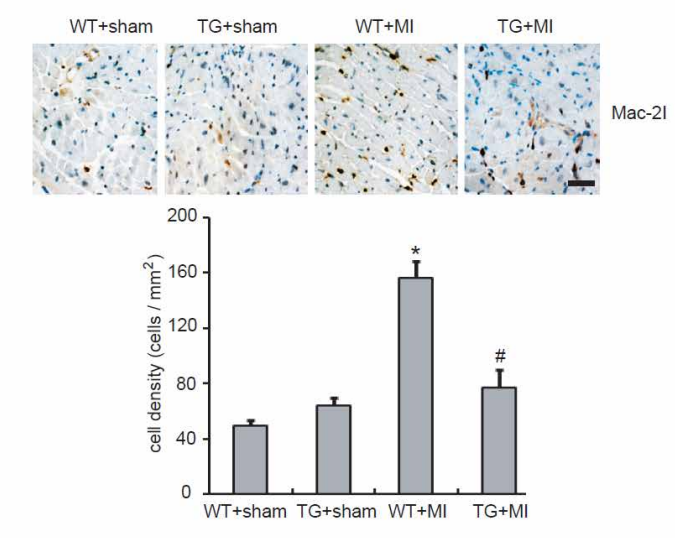

B

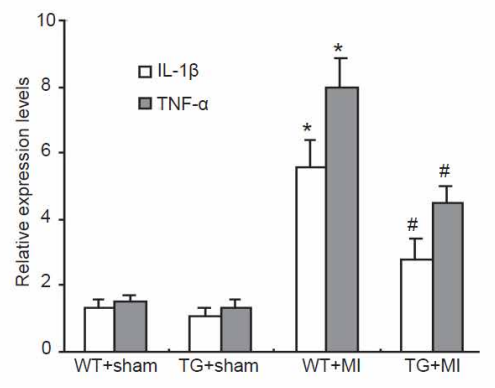

Overexpression of CHIP attenuates myocardial inflammation

Since inflammation is associated with cardiac dysfunction after MI. We then examined whether CHIP regulates cardiac inflammation. As shown in Fig. 4A, the infiltration of Mac-2 positive macrophages was markedly increased in WT hearts but was attenuated in TG mice after MI, (Fig. 4A). Moreover, the mRNA expression of TNF- $\alpha$ and IL-1 $\beta$ was significantly lower in TG than in WT hearts (Fig. 4B).

\section{CHIP overexpression enhances angiogenesis}

Angiogenesis is known to be critical in preserving cardiac function in response to ischemic stress [17]. To determine the effects of CHIP overexpression on angiogenesis after MI, we examined the formation of capillary and arteriole in the heart tissue by isolectin and immunohistochemical staining. The border zone capillary density and $\alpha$-SMA positive cells (a marker of smooth muscle cells) was markedly decreased in infarcted WT heart compared with shamed hearts but was maintained in TG heart after MI (Fig. 5A and B). There were similar alterations between 2 groups after sham (Fig. 5A and B). These data suggest that angiogenesis was increased in TG mice.

CHIP overexpression down-regulates $p 53$ protein level and up-regulates HIF-1 $\alpha$ and VEGF expression

The tumour suppressor p53 is a central transcriptional regulator of multiple proapoptotic and angiogenic genes [26], which is known to be a downstream target of CHIP [16]. We therefore examined the effect of CHIP overexpression on the level of p53 protein and the expression of its downstream targets such as HIF- $1 \alpha$ and VEGF expression. In fact, Western blotting of myocardial extracts showed a marked increase of p53 protein expression and decrease of HIF- $1 \alpha$ in WT heart after MI. In contrast, this effect was markedly reversed in TG heart (Fig. 5C). Moreover, CHIP overexpression significantly enhanced the expression of both HIF- $1 \alpha$ and VEGF mRNA compared with WT heart after MI (Fig. 5D), in accordance 
A
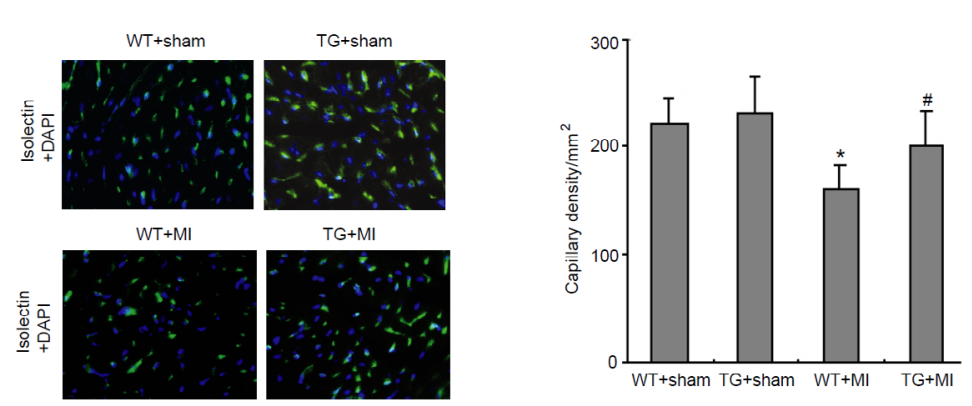

B

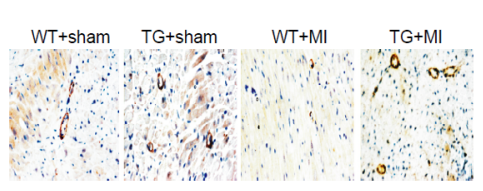

C
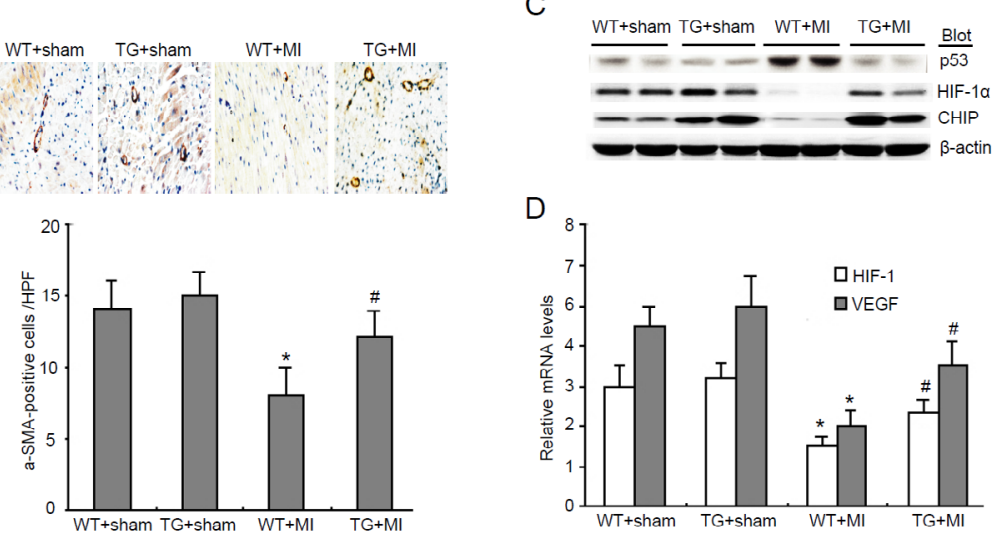

Fig. 5. CHIP overexpression enhances angiogenesis. (A) Representative fluorescein isothiocyanateconjugated isolectin staining of myocardial capillaries (left). Green areas represent isolectin-positive cells, and blue is DAPI for nuclei. Bar graph shows the quantification of capillary density from WT and TG mice at 4 weeks after sham and myocardial infarction (MI). (B) Immunohistochemical staining with anti-a-smooth muscle actin (a-SMA) antibody in the border zone of the heart at 4 weeks after sham and myocardial infarction (MI) (left). Bar graph shows the quantification of a-SMA-positive cells. (C) Western blot analysis of expression of p53 and HIF-1 $\alpha$ protein from the border zone of the heart at 4 weeks after sham and myocardial infarction (MI). (D) qPCR analysis of the mRNA levels of HIF1 and VEGF in the myocardial border area from WT and TG mice after sham and myocardial infarction (MI). Data expressed as means $\pm \mathrm{S}$. E. M. (n=8 per group). ${ }^{*} P<0.05$ vs. sham; ${ }^{\#} P<0.05$ vs. WT + MI.

with an increase in capillary and arteriole densities in TG mice (Fig. 5A and B). These results establish that CHIP exerts important anti-apoptotic effects and also promotes angiogenesis in the infarcted heart through down-regulation of p53 protein.

\section{Discussion}

In the present study, we demonstrated that cardiac-specific overexpression of CHIP enhanced angiogenesis, reduced infarct size, cardiomyocyte apoptosis and inflammation, improved cardiac dysfunction and survival in mice 4 weeks after MI. These results expanded on previous data, and provided the first in vivo evidence for the critical role of CHIP in prevention of cardiac remodeling and dysfunction 4 weeks after MI. The mechanisms were associated with reduction of p53-induced apoptosis, inhibition of inflammation and enhancement of HIF-1 $\alpha$ /VEGF-dependent angiogenesis. 
Numerous studies have demonstrated that E3 ubiquitin ligases play important roles in protecting myocardial infarction, cardiac hypertrophy and fibrosis $[15,16,18,24,25$, 27-29]. CHIP has been identified as an E3 ubiquitin ligase/chaperone protein that is highly expressed in the various tissues and cell types [26]. To date, CHIP is reported to target over 10 putative substrates thereby regulating cell growth, differentiation, apoptosis, inflammation and neurodegeneration $[8,11,13-17,30]$. Recent studies indicate that CHIP has a critical role in preserving cardiomyocyte death through degradation of apoptosis signal-regulated kinase 1 (ASK1) and p53, which are known to promote cardiomyocyte apoptosis in response to ischaemia/reperfusion (I/R) and MI injury [11, 15-17]. Consistent with these results, our data demonstrated that in addition to inhibiting cardiomyocyte apoptois, increased expression of CHIP also attenuated inflammation, enhanced angiogenesis, leading to improving cardiac dysfunction and survival 4 weeks after MI (Fig. 1, 2, 3).

There are various studies indicating a well relationship between cardiac angiogenesis and cardiac function $[2,3]$. Angiogenesis is an important repair mechanism in response to ischaemia/reperfusion (I/R) injury through increasing blood flow and oxygen supply [2,3]. Previous studies suggested that many factors are responsible for promoting the angiogenesis process during cardiac ischaemia, including haemodynamics, hypoxia, inflammation and angiogenic factors $[2,3]$. Recently, p53/HIF-1 $\alpha$ /VEGF signaling pathway has been demonstrated to plays important roles in the neovascularization response in pathological conditions like ischaemia and pressure-overload [2, 26, 31]. In addition to its role in apoptosis, p53 also play an important role in angiogenesis through inhibition of HIF-1 $\alpha$ transcriptional activity and VEGF expression [26]. Moreover, cardiomyocyte specific VEGF knockout mice exhibit decreased capillary density and contractile dysfunction. Treatment with a decoy VEGF receptor attenuates angiogenesis during cardiac growth. However, whether CHIP affects p53-mediated HIF-1 $\alpha$ /VEGF signaling and cardiac angiogenesis after infarction remain to be defined. The present study provided the first in vivo evidence, to our knowledge, demonstrated that sustained CHIP expression in heart markedly reduced p53 expression and increased HIF- $1 \alpha$ and VEGF expression leading to enhanced angiogenesis. Thus, our study in CHIP transgenic mice suggest that further enhancement of CHIP level may be a novel therapeutic strategy to prevent progression of heart failure after infarction. The important mechanism of these beneficial effects appears to be partly the inhibition of p53mediated anti-angiogenic effects.

In summary, the studies reported herein reveal a novel mechanism for CHIP to improve cardiac dysfunction after MI. Our results establish that overexpression of CHIP plays an important beneficial role in counteracting LV remodeling after MI by decreasing cardiomyocyte death, inflammation and fibrosis. We have also demonstrated that this effect is related, at least in part, to enhancing angiogenesis via activation of HIF-1 $\alpha$ /VEGFmediated signaling pathway. Thus, these results suggest increased expression of CHIP should be explored as a therapeutic approach to limit pathological LV remodeling in after MI.

\section{Acknowledgements}

This work was supported by grants from China National Natural Science Funds for Distinguished Young Scholars (No. 81025001), Chang Jiang Scholar Program, the 973 program (No. 2012CB517802) and the Beijing high-level talents program (PHR20110507).

\section{References}

1 Baines CP, Molkentin JD: Stress signaling pathways that modulate cardiac myocyte apoptosis. J Mol Cell Cardiol 2005;38:47-62. 
- Tabibiazar R, Rockson SG: Angiogenesis and the ischaemic heart. Eur Heart J 2001;22:903-918.

3 Syed IS, Sanborn TA, Rosengart TK: Therapeutic angiogenesis: A biologic bypass. Cardiology 2004;101:131143.

4 Sun J, Sui X, Bradbury JA, Zeldin DC, Conte MS, Liao JK: Inhibition of vascular smooth muscle cell migration by cytochrome p450 epoxygenase-derived eicosanoids. Circ Res 2002;90:1020-1027.

-5 Okubo S, Wildner O, Shah MR, Chelliah JC, Hess ML, Kukreja RC: Gene transfer of heat-shock protein 70 reduces infarct size in vivo after ischemia/reperfusion in the rabbit heart. Circulation 2001;103:877-881.

6 Galigniana MD, Harrell JM, Housley PR, Patterson C, Fisher SK, Pratt WB: Retrograde transport of the glucocorticoid receptor in neurites requires dynamic assembly of complexes with the protein chaperone hsp90 and is linked to the chip component of the machinery for proteasomal degradation. Brain Res Mol Brain Res 2004;123:27-36.

7 Meacham GC, Patterson C, Zhang W, Younger JM, Cyr DM: The hsc70 co-chaperone chip targets immature cftr for proteasomal degradation. Nat Cell Biol 2001;3:100-105.

-8 Dickey CA, Kamal A, Lundgren K, Klosak N, Bailey RM, Dunmore J, Ash P, Shoraka S, Zlatkovic J, Eckman CB, Patterson C, Dickson DW, Nahman NS Jr, Hutton M, Burrows F, Petrucelli L: The high-affinity hsp90chip complex recognizes and selectively degrades phosphorylated tau client proteins. J Clin Invest 2007;117:648-658.

-9 Al-Ramahi I, Lam YC, Chen HK, de Gouyon B, Zhang M, Perez AM, Branco J, de Haro M, Patterson C, Zoghbi HY, Botas J: Chip protects from the neurotoxicity of expanded and wild-type ataxin-1 and promotes their ubiquitination and degradation. J Biol Chem 2006;281:26714-26724.

10 Alberti S, Bohse K, Arndt V, Schmitz A, Hohfeld J: The cochaperone hspbp1 inhibits the chip ubiquitin ligase and stimulates the maturation of the cystic fibrosis transmembrane conductance regulator. Mol Biol Cell 2004;15:4003-4010.

11 Hwang JR, Zhang C, Patterson C: C-terminus of heat shock protein 70-interacting protein facilitates degradation of apoptosis signal-regulating kinase 1 and inhibits apoptosis signal-regulating kinase 1-dependent apoptosis. Cell Stress Chaperones 2005;10:147-156.

12 Zhou P, Fernandes N, Dodge IL, Reddi AL, Rao N, Safran H, DiPetrillo TA, Wazer DE, Band V, Band H: Erbb2 degradation mediated by the co-chaperone protein chip. J Biol Chem 2003;278:13829-13837.

13 Li F, Xie P, Fan Y, Zhang H, Zheng L, Gu D, Patterson C, Li H: C terminus of hsc70-interacting protein promotes smooth muscle cell proliferation and survival through ubiquitin-mediated degradation of foxo1. J Biol Chem 2009;284:20090-20098.

14 Xie P, Fan Y, Zhang H, Zhang Y, She M, Gu D, Patterson C, Li H: Chip represses myocardin-induced smooth muscle cell differentiation via ubiquitin-mediated proteasomal degradation. Mol Cell Biol 2009;29:23982408.

15 Zhang C, Xu Z, He XR, Michael LH, Patterson C: Chip, a cochaperone/ubiquitin ligase that regulates protein quality control, is required for maximal cardioprotection after myocardial infarction in mice. Am J Physiol Heart Circ Physiol 2005;288:H2836-2842.

16 Naito AT, Okada S, Minamino T, Iwanaga K, Liu ML, Sumida T, Nomura S, Sahara N, Mizoroki T, Takashima A, Akazawa H, Nagai T, Shiojima I, Komuro I: Promotion of chip-mediated p53 degradation protects the heart from ischemic injury. Circ Res 2010;106:1692-1702.

17 Dai Q Zhang C, Wu Y, McDonough H, Whaley RA, Godfrey V, Li HH, Madamanchi N, Xu W, Neckers L, Cyr D, Patterson C: Chip activates hsf1 and confers protection against apoptosis and cellular stress. Embo J 2003;22:5446-5458.

18 Yang K, Zhang TP, Tian C, Jia LX, Du J, Li HH: Carboxyl terminus of heat shock protein 70-interacting protein inhibits angiotensin ii-induced cardiac remodeling. Am J Hypertens 2012;25:994-1001.

19 Gao E, Lei YH, Shang X, Huang ZM, Zuo L, Boucher M, Fan Q Chuprun JK, Ma XL, Koch WJ: A novel and efficient model of coronary artery ligation and myocardial infarction in the mouse. Circ Res 2010;107:1445-1453.

20 Zhang Y, Kang YM, Tian C, Zeng Y, Jia LX, Ma X, Du J, Li HH: Overexpression of nrdp1 in the heart exacerbates doxorubicin-induced cardiac dysfunction in mice. PLoS One 2011;6:e21104.

21 Li Y, Zhang C, Wu Y, Han Y, Cui W, Jia L, Cai L, Cheng J, Li H, Du J: Interleukin-12p35 deletion promotes cd4 t-cell-dependent macrophage differentiation and enhances angiotensin ii-induced cardiac fibrosis. Arterioscler Thromb Vasc Biol 2012;32:1662-1674. 


\section{Cellular Physiology $\quad$ Cell Physiol Biochem 2013;31:199-208

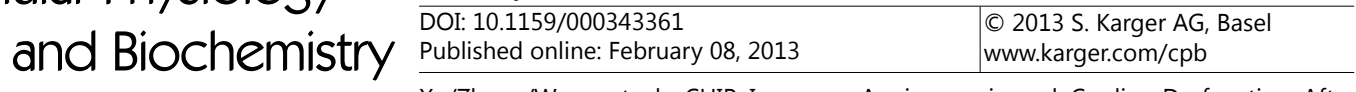 \\ Xu/Zhang/Wang et al.: CHIP Improves Angiogenesis and Cardiac Dysfunction After Infarction}

22 Yang M, Zheng J, Miao Y, Wang Y, Cui W, Guo J, Qiu S, Han Y, Jia L, Li H, Cheng J, Du J: Serum-glucocorticoid regulated kinase 1 regulates alternatively activated macrophage polarization contributing to angiotensin ii-induced inflammation and cardiac fibrosis. Arterioscler Thromb Vasc Biol 2012;32:1675-1686.

23 Zhang Y, Zeng Y, Wang M, Tian C, Ma X, Chen H, Fang Q Jia L, Du J, Li H: Cardiac-specific overexpression of e3 ligase nrdp1 increases ischemia and reperfusion-induced cardiac injury. Basic Res Cardiol 2011;106:371-383.

24 Li HH, Kedar V, Zhang C, McDonough H, Arya R, Wang DZ, Patterson C: Atrogin-1/muscle atrophy f-box inhibits calcineurin-dependent cardiac hypertrophy by participating in an scf ubiquitin ligase complex. J Clin Invest 2004;114:1058-1071.

25 Li HH, Willis MS, Lockyer P, Miller N, McDonough H, Glass DJ, Patterson C: Atrogin-1 inhibits akt-dependent cardiac hypertrophy in mice via ubiquitin-dependent coactivation of forkhead proteins. J Clin Invest 2007;117:3211-3223.

26 Sano M, Minamino T, Toko H, Miyauchi H, Orimo M, Qin Y, Akazawa H, Tateno K, Kayama Y, Harada M, Shimizu I, Asahara T, Hamada H, Tomita S, Molkentin JD, Zou Y, Komuro I: P53-induced inhibition of hif-1 causes cardiac dysfunction during pressure overload. Nature 2007;446:444-448.

27 Hu S, Liu S, Zheng Z, Yuan X, Li L, Lu M, Shen R, Duan F, Zhang X, Li J, Liu X, Song Y, Wang W, Zhao S, He Z, Zhang H, Yang K, Feng W, Wang X: Isolated coronary artery bypass graft combined with bone marrow mononuclear cells delivered through a graft vessel for patients with previous myocardial infarction and chronic heart failure: A single-center, randomized, double-blind, placebo-controlled clinical trial. J Am Coll Cardiol 2011;57:2409-2415.

28 Usui S, Maejima Y, Pain J, Hong C, Cho J, Park JY, Zablocki D, Tian B, Glass DJ, Sadoshima J: Endogenous muscle atrophy f-box mediates pressure overload-induced cardiac hypertrophy through regulation of nuclear factor-kappab. Circ Res 2011;109:161-171.

29 Zhang XH, Li SC, Xie F, Lo NN, Yang KY, Yeo SJ, Fong KY, Thumboo J: An exploratory study of response shift in health-related quality of life and utility assessment among patients with osteoarthritis undergoing total knee replacement surgery in a tertiary hospital in singapore. Value Health 2012;15:S72-78.

30 Esser C, Scheffner M, Hohfeld J: The chaperone-associated ubiquitin ligase chip is able to target p53 for proteasomal degradation. J Biol Chem 2005;280:27443-27448.

-31 Song H, Yin D, Liu Z: Gdf-15 promotes angiogenesis through modulating p53/hif-1alpha signaling pathway in hypoxic human umbilical vein endothelial cells. Mol Biol Rep 2012;39:4017-4022. 